\title{
Vector boson plus one jet production in POWHEG
}

\author{
Simone Alioli, ${ }^{a, b}$ Paolo Nason, ${ }^{b}$ Carlo Oleari ${ }^{b, c}$ and Emanuele $\mathbf{R e}^{b, d}$ \\ ${ }^{a}$ Deutsches Elektronen-Synchrotron DESY, \\ Platanenallee 6, D-15738 Zeuthen, Germany \\ ${ }^{b}$ INFN, Sezione di Milano-Bicocca, \\ Piazza della Scienza 3, 20126 Milan, Italy \\ ${ }^{c}$ Università di Milano-Bicocca, \\ Piazza della Scienza 3, 20126 Milan, Italy \\ ${ }^{d}$ Institute for Particle Physics Phenomenology, Department of Physics, \\ University of Durham, Durham, DH1 3LE, U.K. \\ E-mail: simone.alioli@desy.de, Paolo.Nason@mib.infn.it, \\ Carlo.0leari@mib.infn.it, emanuele.re@durham.ac.uk
}

ABSTRACT: We present an implementation of the next-to-leading order vector boson plus one jet production process in hadronic collision in the framework of POWHEG, which is a method to implement NLO calculations within a Shower Monte Carlo context. All spin correlations in the vector boson decay products have been taken into account. The process has been implemented in the framework of the POWHEG BOX, an automated computer code for building implementations of NLO calculations that can be interfaced to a shower Monte Carlo program. We present phenomenological results for the case of the $Z / \gamma$ plus one jet production process, obtained by matching the POWHEG calculation with the shower performed by PYTHIA, for the LHC, and we compare our results with available Tevatron data.

KeYwords: Jets, NLO Computations, Hadronic Colliders, QCD

ARXIV EPRINT: 1009.5594 


\section{Contents}

1 Introduction $\quad 1$

2 Description of calculation $\quad 2$

2.1 Generation cut and Born suppression factor 4

$\begin{array}{lll}2.2 & \text { Negative-weight events } & 6\end{array}$

$\begin{array}{lll}3 & \text { Validation of the generated samples } & \mathbf{7}\end{array}$

3.1 Negative weights and folding 8

3.2 Comparison with MCFM 9

$\begin{array}{llr}4 & \text { Phenomenology } & 10\end{array}$

$\begin{array}{lll}\text { 4.1 CDF results } & 10\end{array}$

$\begin{array}{lll}4.2 \text { D0 results } & 13\end{array}$

$\begin{array}{lll}4.3 & \text { LHC results } & 17\end{array}$

$\begin{array}{llr}5 & \text { Conclusions } & 18\end{array}$

$\begin{array}{lr}\text { A Tracking negative-weight events } & 19\end{array}$

B Negative weights and folding $\quad 20$

\section{Introduction}

The process of vector boson production in association with jets plays an important role at present hadron colliders. In the early LHC phase, $Z$ plus one jet production will play a major role in jet calibration. Furthermore, vector boson production in association with jets is an important background to new physics signals. In particular, when the $Z$ decays into neutrinos, it becomes a source of missing-energy signals. The analogous process of $W$ production, in association with jets, gives origin to an important background to the production of a lepton in association with missing energy and jets.

$Z$ plus one jet production has a very distinctive signature, when the $Z$ decays into muons or electrons, and several studies have been performed at the Tevatron, both by the CDF [1] and by the D0 Collaboration [2-5]. They are all carried out by correcting the measured quantities to the particle level, according to the recommendations developed in the 2007 Les Houches workshop [6], and then compared to theoretical calculations, computed mostly at the next-to-leading order (NLO) level, and corrected for showering, hadronization and underlying-event effects. These corrections, in turn, are extracted from shower Monte Carlo (SMC) programs. 
In the present work, we present a calculation for the NLO cross section for vector boson plus one jet that can be interfaced to a shower Monte Carlo program, within the POWHEG framework $[7,8]$. This is the first time that such calculation has been performed. More specifically, we have implemented this process using the POWHEG BOX, a general computer code framework for embedding NLO calculations in shower Monte Carlo programs according to the POWHEG method. In fact, the POWHEG BOX framework was developed using the $Z / \gamma+1 j$ process as its first testing example. ${ }^{1}$

It is clear that, by using the POWHEG implementation of $V+1 j$ production, the comparison of the theoretical prediction with the experimental results is eased considerably, and is also made more precise. Rather than estimating shower and underlying-event corrections using a parton shower program, one interfaces directly the parton shower to the hard process in question, yielding an output that can be compared to the experimental results at the particle level. We will carry out this task for the $Z+1 j$ case, and compare our results to the Tevatron findings. We remark, however, that a further improvement to this study could be carried out, by using the POWHEG program to generate the events that are fed through the detector simulation, and are directly compared to raw data. We are not, of course, in a position to perform such a task, that should instead be carried out by the experimental collaborations.

The paper is organized as follows. In section 2 we give a brief description of our calculation. Since we used the POWHEG BOX to implement our process, we refer the reader to the POWHEG BOX publication [9], and report here only a few details that are particularly relevant to the process in question. In the following, we always consider the $Z+1 j$ case, since the $W+1 j$ is fully analogous. In section 3 we describe the generation of the $Z+1 j$ sample, together with some consistency checks of our calculation, and new features added to the POWHEG BOX. In section 4, we present results for the Tevatron and for the LHC at $14 \mathrm{TeV}$. Comparison with available data from the CDF and D0 Collaborations are carried out. Finally, in section 5 , we give our final remarks.

\section{Description of calculation}

We have considered the hadroproduction of a single vector boson plus one jet at NLO order, with all spin correlations from the decay taken into account. All fermions (quarks and leptons) have been treated in the massless limit (no top-quark contributions have been taken into account).

In order to implement a new process at NLO into the POWHEG BOX, we have to provide the following ingredients:

i. The list of all flavour structures of the Born processes.

ii. The list of all flavour structures of the real processes.

\footnotetext{
${ }^{1}$ In the rest of the paper, for ease of notation, we will refer to the $Z / \gamma+1 j$ process simply as " $Z+1 j$ production", without mentioning the presence of the photon, whose effects, together with all the spin correlations of the decay products, have been fully taken into account.
} 


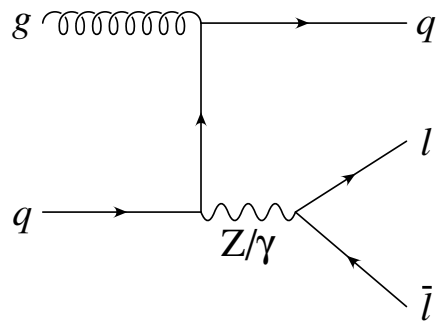

(a) Born

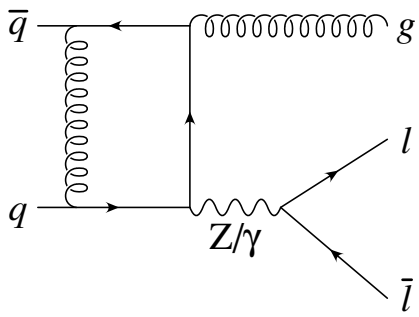

(d) virtual

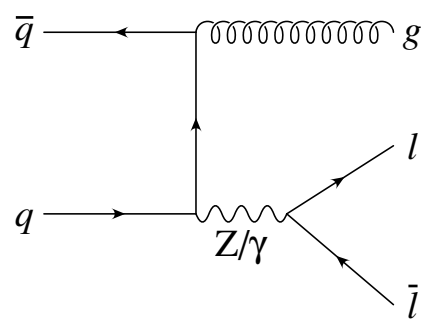

(b) Born

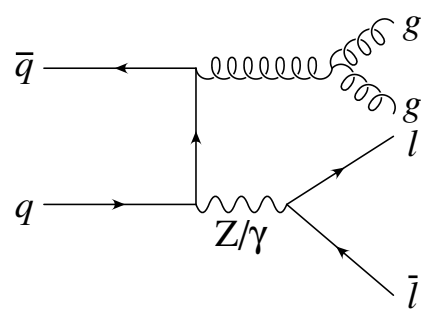

(e) real

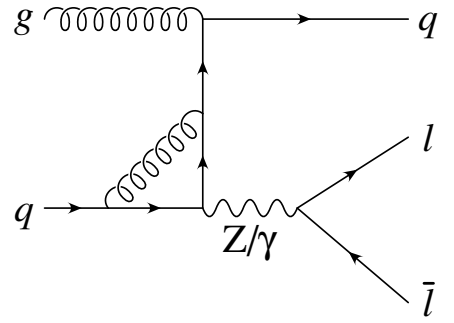

(c) virtual

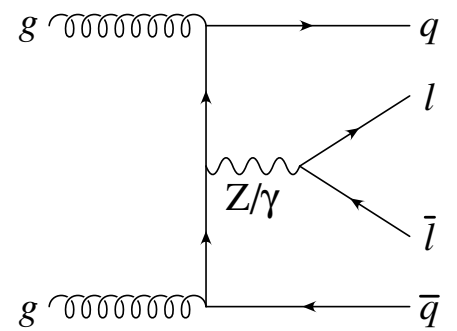

(f) real

Figure 1. Sample graphs for the Born, virtual and real contributions to the $Z / \gamma+1 j$ production process.

iii. The Born phase space.

iv. The real matrix elements squared for all relevant partonic processes.

v. The finite part of the virtual corrections computed in dimensional regularization or in dimensional reduction.

vi. The Born squared amplitudes $\mathcal{B}$, the colour correlated ones $\mathcal{B}_{i j}$ and spin correlated ones $\mathcal{B}_{\mu \nu}$.

vii. The Born colour structures in the limit of a large number of colours.

For the case at hand, the list of processes is generated going through all possible massless quarks and gluons that are compatible with the production of the vector boson plus an extra parton.

The Born phase space for this process poses no challenges: we generate the momentum of the vector boson distributed according to a Breit-Wigner function, plus one extra light particle. The vector boson momentum is then further decayed into two momenta, describing the final-state leptons. At this stage, the momentum fractions $x_{1}$ and $x_{2}$ are also generated and the momenta of the incoming partons are computed.

A sample of Feynman diagrams that contribute to the $Z+1 j$ process at the Born level $(\mathcal{B})$ is depicted in panels $(\mathrm{a})$ and $(\mathrm{b})$ of figure 1 . Together with the Born diagrams, we have to consider the one-loop corrections to the tree level graphs, and the diagrams 


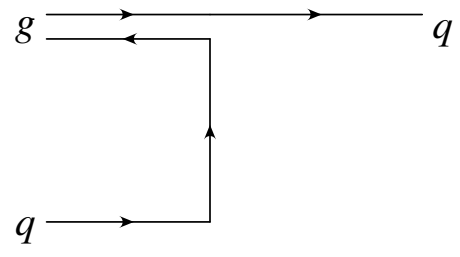

(a)

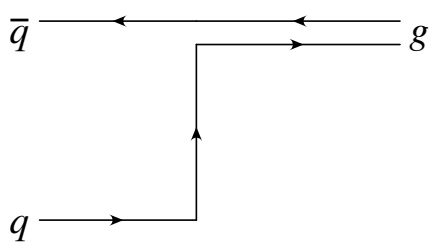

(b)

Figure 2. Born colour structures of the two Feynman diagrams illustrated in panels (a) and (b) in figure 1.

with an extra radiated parton. A sample of virtual and real contributions is depicted in panels (c)-(f) of figure 1 .

We have computed the Born and real contributions ourselves, using the helicityamplitude technique of refs. $[10,11]$. The amplitudes are computed numerically in a fortran code, as complex numbers, and squared at the end. The finite part of the virtual corrections has been taken from the MCFM program [12], that uses the virtual matrix elements given in ref. [13] and computed first in ref. [14].

The use of the helicity-amplitude technique to compute the amplitudes at the Born level facilitates the calculation of the spin-correlated matrix elements $\mathcal{B}_{\mu \nu}$. In fact, they are the Born amplitudes just before being numerically contracted with the polarization vector of the initial- or final-state gluon.

Since we are dealing with three coloured partons at the Born level, the colour correlated Born amplitudes $\mathcal{B}_{i j}$ are all proportional to the Born one. The two independent colourcorrelated matrix elements are given by

$$
B_{q q^{\prime}}=\frac{1}{2}\left(2 C_{\mathrm{F}}-C_{\mathrm{A}}\right) \mathcal{B}, \quad B_{q g}=\frac{C_{\mathrm{A}}}{2} \mathcal{B},
$$

and, from colour conservation, they satisfy

$$
\sum_{i, i \neq j} \mathcal{B}_{i j}=C_{f_{j}} \mathcal{B}
$$

where $i$ runs over all coloured particles entering or exiting the process, and $C_{f_{j}}$ is the Casimir constant for the colour representation of particle $j$.

Finally, the Born colour structures, in the limit of a large number of colours, are straightforward, due to the presence of a single gluon and two quarks at the Born level. For the two Born diagrams depicted in figure 1, the colour structures are shown in figure 2.

\subsection{Generation cut and Born suppression factor}

The $V+1 j$ process differs substantially from all processes previously implemented in POWHEG, in the fact that the Born contribution itself is collinear and infrared divergent. In all previous implementations, the Born diagrams were finite, and it was thus possible to generate an unweighted set of underlying Born configurations covering the whole phase 
space. In the present case, this is not possible, since they would all populate the very low transverse momentum region. Of course, this problem is also present in standard shower Monte Carlo programs, where it is dealt with by generating the Born configuration with a cut $k_{\text {gen }}$ on the transverse momentum of the $V$ boson. After the shower, one must discard all events that fail some transverse momentum analysis cut $k_{\text {an }}$ in order to get a realistic sample. The analysis cut $k_{\text {an }}$ may be applied to the transverse momentum of the vector boson, or to the hardest jet. We assume here, for sake of discussion, that it is applied to the vector boson transverse momentum.

Taking $k_{\text {an }} \gtrsim k_{\text {gen }}$ is not enough to get a realistic sample. In fact, in an event generated at the Born level with a given $k_{\mathrm{T}}<k_{\text {gen }}$, the shower may increase the transverse momentum of the jet so that the final vector-boson transverse momentum $k_{\mathrm{T}}^{V}$ can be bigger than $k_{\mathrm{an}}$. Thus, even if the generation cut is below the analysis cut, it may reduce the number of events that pass the analysis cut. Of course, as we lower $k_{\text {gen }}$ keeping $k_{\text {an }}$ fixed, we will reach a point where very few events below $k_{\text {gen }}$ will pass the analysis cut $k_{\text {an }}$. In fact, generation of radiation with transverse momentum larger than $k_{\text {gen }}$ is strongly suppressed in POWHEG, and, in turn, radiation from subsequent shower is required to be not harder than the hardest radiation of POWHEG. Thus, if we want to generate a sample with a given $k_{\text {an }}$ cut, we should choose $k_{\text {gen }}$ small enough, so that the final sample remains substantially the same if $k_{\text {gen }}$ is lowered even further.

Together with the option of generating events with a generation cut $k_{\text {gen }}$, a second option for the implementation of processes with a divergent Born contribution is also available. It requires that we generate weighted events, rather than unweighted ones. This is done by using a suppressed cross section for the generation of the underlying Born configurations

$$
\bar{B}_{\text {supp }}=\bar{B} \times F\left(k_{\mathrm{T}}\right),
$$

where $\bar{B}$ is the inclusive NLO cross section at fixed underlying Born variables, $k_{\mathrm{T}}$ is the transverse momentum of the vector boson in the underlying Born configuration and $F\left(k_{\mathrm{T}}\right)$ is a function that goes to zero in the $k_{\mathrm{T}} \rightarrow 0$ limit fast enough to make $\bar{B} \times F\left(k_{\mathrm{T}}\right)$ finite in this limit. In this way, $\bar{B}_{\text {supp }}$ is integrable, and one can use it to generate underlying Born configurations according to its value. The generated events, however, should be given a weight $1 / F\left(k_{\mathrm{T}}\right)$, rather than 1 , in order to compensate for the initial $F\left(k_{\mathrm{T}}\right)$ suppression factor. With this method, events do not concentrate in the low $k_{\mathrm{T}}$ region, although their weight, in the low $k_{\mathrm{T}}$ region, becomes divergent. After shower, if one imposes the analysis cut, one gets a finite cross section, since it is unlikely that events with small transverse momentum at the Born level may pass the cut after shower.

In recent POWHEG BOX revisions, both methods can be implemented at the same time. We wanted in fact to be able to implement the following three options:

- Generate events using a transverse momentum generation cut $k_{\text {gen }}$.

- Generate events using a Born suppression factor, and a small transverse momentum cut, just enough to avoid unphysical values of the strong coupling constant and of the factorization scale that appears in the parton distribution functions. In this case, 
since we are also generating events with very small transverse momenta, radiative corrections may become larger than the Born term, and negative-weight events could be generated. We should then be prepared to track them. This issue will be further discussed in the next section.

- Apply a Born suppression factor, and set the transverse momentum cut $k_{\text {gen }}$ to zero. In this case the program cannot be used to generate events. It can be used, however, to produce NLO fixed order distributions, provided the renormalization and factorization scales are set in such a way that they remain large enough even at small transverse momentum $k_{\mathrm{T}}^{V}$. This feature is only used for the generation of fixed-order distributions.

The generation cut is activated by setting the token bornktmin to the desired value in the powheg.input file. The Born suppression is activated by setting the token bornsuppfact to a positive real value. The process-specific subroutine born_suppression sets the suppression factor of eq. (2.3) to

$$
F\left(k_{\mathrm{T}}\right)=\frac{k_{\mathrm{T}}^{2}}{k_{\mathrm{T}}^{2}+\text { bornsuppfact }^{2}} .
$$

If bornsuppfact is negative, the suppression factor is set to 1 .

The need of a transverse momentum cut is not only a technical issue. The NLO calculation of $V+1 j$ production holds only if the transverse momentum of the vector boson is not too small. In fact, as the $k_{\mathrm{T}}$ decreases, large Sudakov logarithms arise in the NLO correction, and the value of the running coupling increases, up to the point where the cross section, at fixed order, becomes totally unreliable. These large logarithms should all be resummed in order to get a sensible answer in this region. In the POWHEG implementation of single vector-boson production [15], in fact, these logarithms are all resummed. Then it is clear that some sort of merging between the $V+1 j$ and the $V$ production processes should be performed at relatively small transverse momentum, in order to properly deal with this problem. Here we will not attempt to perform such merging, that we leave for future work. We will simply recall, when looking at our results, that we expect to get unphysical distributions when the vector boson transverse momentum is too small. We will discuss this fact in a more quantitative way in section 3.

\subsection{Negative-weight events}

In the POWHEG method, negative-weight events can only arise if one is approaching a region where the NLO computation is no longer feasible. In our study for the $V+1 j$ process, we approach this region at small transverse momenta. In order to better understand what happens there, rather than neglecting negative weights (that is the default behaviour of the POWHEG BOX), we have introduced a new feature in the program, that allows one to track also the negative-weight events. Since this is a technical feature, we describe at length it in appendix A. 

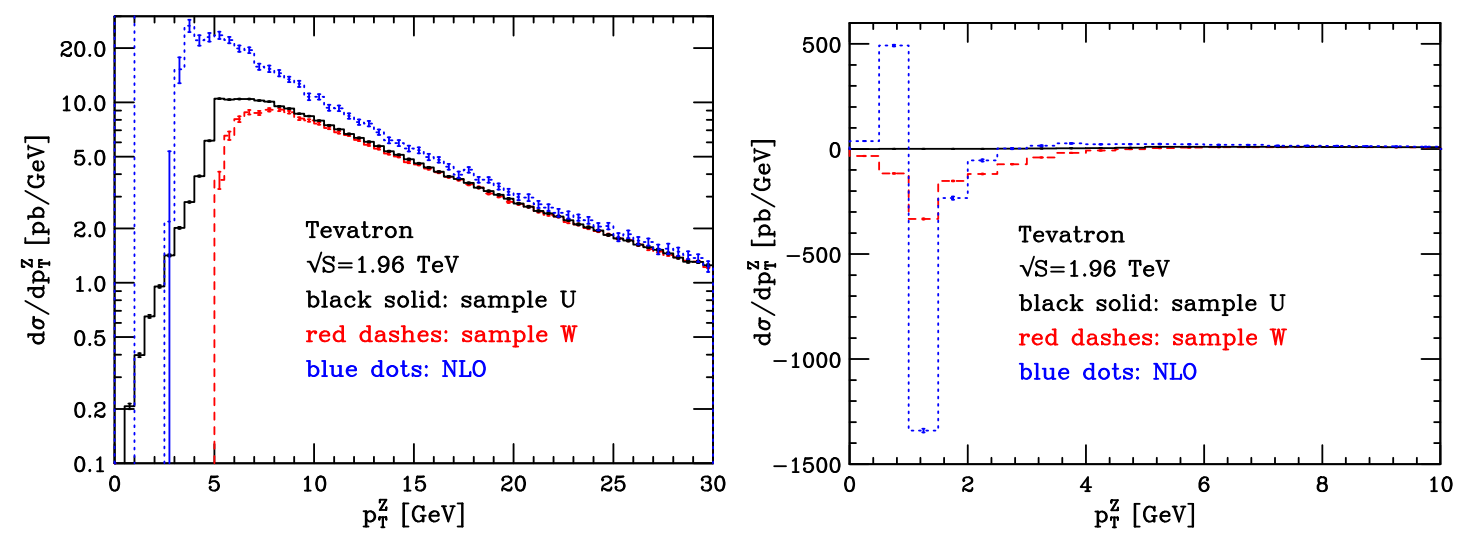

Figure 3. $Z$ transverse momentum in samples $\mathrm{U}, \mathrm{W}$ and at fixed NLO order.

\section{Validation of the generated samples}

In this section we discuss the output of the POWHEG BOX for $Z+1 j$ production. We consider here $p \bar{p}$ collisions at $1.96 \mathrm{TeV}$. We use the CTEQ6M pdf set [16]. The factorization and renormalization scales (in the computation of the $\bar{B}$ function) are fixed to the transverse momentum of the vector boson of the underlying Born configuration. For the generation of radiation, these scales are set by the POWHEG BOX according to the prescriptions given in [9], i.e. they are set equal to the transverse momentum of the produced radiation. More precisely, in case of initial-state radiation, the scale is set to the transverse component of the momentum of the radiated parton relative to the collision axis. In case of final-state radiation, one uses instead $2 E^{2}(1-\cos \theta)$, where $E$ is the energy of the radiated parton in the partonic center of mass, and $\theta$ is the angle between the radiated parton and the parton that has emitted it (i.e. it is the splitting angle). For small angles, this last choice also coincides with the transverse momentum relative to the emitting parton. It has, however, the advantage that, for large angles approaching backward emission, it does not vanish, a desirable feature in the case of final-state radiation.

We have first produced one sample with a generation cut $k_{\text {gen }}=5 \mathrm{GeV}$ on the transverse momentum of the $Z$ boson in the underlying Born configuration. This sample, that we call sample U (for unweighted), was produced with positive weights. Then we produced a second sample W, where we used a Born suppression factor, with bornsuppfact $=10 \mathrm{GeV}$, and a generation cut of $1 \mathrm{GeV}$, in order to avoid unphysical values for the strong coupling and pdf's (we will assume, in the following, that this tiny generation cut has no effects on distributions where the $Z$ boson or the hardest jet have transverse momenta of several $\mathrm{GeV}$ ). We have also set withnegweights to 1 , so that we are able to record negative weighted events that may arise in the region of very small transverse momenta. Sample W is thus weighted, with weights of either signs. We analyzed the events of the two samples at the POWHEG level, i.e. without feeding them to a shower Monte Carlo. We begin by comparing the transverse momentum distribution of the $Z$ of the two samples, and of the fixed order NLO QCD result (obtained as a byproduct of the event generation) in figure 3 . The 

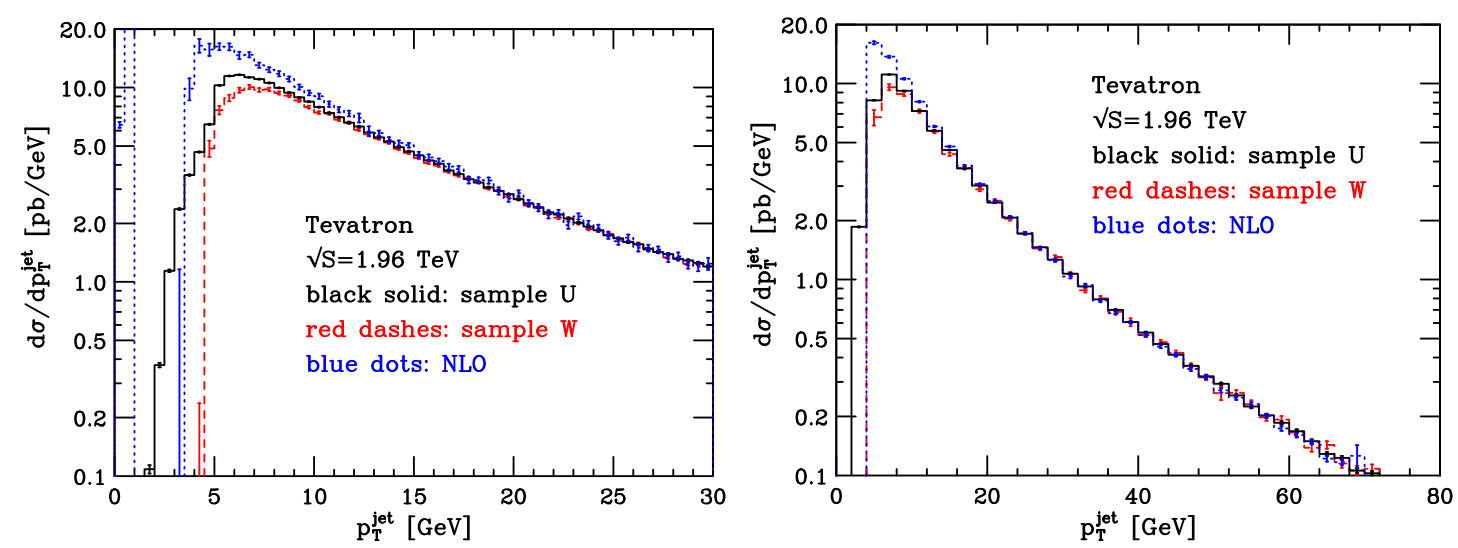

Figure 4. Transverse momentum of the hardest jet in samples $U$ and $W$.

figure should be interpreted in the following way. The weighted sample gives the reference result, since it is unaffected by the generation cut. The U sample feels clearly the effect of the $5 \mathrm{GeV}$ generation cut. However, for $p_{T}^{Z} \gtrsim 10 \mathrm{GeV}$, the $\mathrm{U}$ and $\mathrm{W}$ results coincide, showing that the effect of the generation cut in this region is fully negligible. A second important observation has to do with results in the $\mathrm{W}$ sample. It becomes unphysical for $p_{T}^{Z} \lesssim 5 \mathrm{GeV}$. This indicates that NLO corrections in this region are out of control, and that our program should not be used for $p_{T}^{Z} \lesssim 10 \mathrm{GeV}$. The fixed order NLO result displays a similarly unphysical behaviour in the low transverse momentum region. The similarity is more apparent in the right panel of figure 3, which is given in linear scale. There is a very large negative value of the NLO cross section in the bin between 1 and $1.5 \mathrm{GeV}$. The POWHEG result follows the NLO result up to a certain amount of smearing.

Plots for the transverse momentum of the hardest jet are shown in figure 4 . They are similar to the plots in the $Z$ transverse momentum, except for a more modest value of the NLO distribution at small transverse momenta.

Finally, in figure 5, the rapidity distribution of the $Z$ and of the hardest jet are plotted for the $\mathrm{U}$ and $\mathrm{W}$ sample and for the NLO contribution. A cut of $10 \mathrm{GeV}$ is applied on either the $Z$ or the jet transverse momentum. We see, again, very good agreement between the $\mathrm{U}$ and $\mathrm{W}$ samples at this transverse momentum. We have also analyzed many other distributions for the $\mathrm{U}$ and $\mathrm{W}$ sample. They all lead to the conclusion that, when cuts on the transverse momenta of the order of $10 \mathrm{GeV}$ (twice the generation cut) are applied, the $\mathrm{U}$ sample yields distributions that are equivalent to the $\mathrm{W}$ sample. More specifically, at $10 \mathrm{GeV}$, in the differential cross section of the jet or of the $Z$ transverse momentum, the $\mathrm{U}$ sample differs from the $\mathrm{W}$ sample by $6 \%$, and the difference decreases with $p_{\mathrm{T}}$. Thus, we conclude that for $p_{\mathrm{T}}>10 \mathrm{GeV}$, at Tevatron energies, the $\mathrm{U}$ sample is substantially independent of a generation cut less or equal than $5 \mathrm{GeV}$.

\subsection{Negative weights and folding}

In order to get a reasonably small fraction of negative-weight events in the POWHEG BOX, it is at times necessary to increase the folding parameters foldcsi, foldy and foldphi. These 

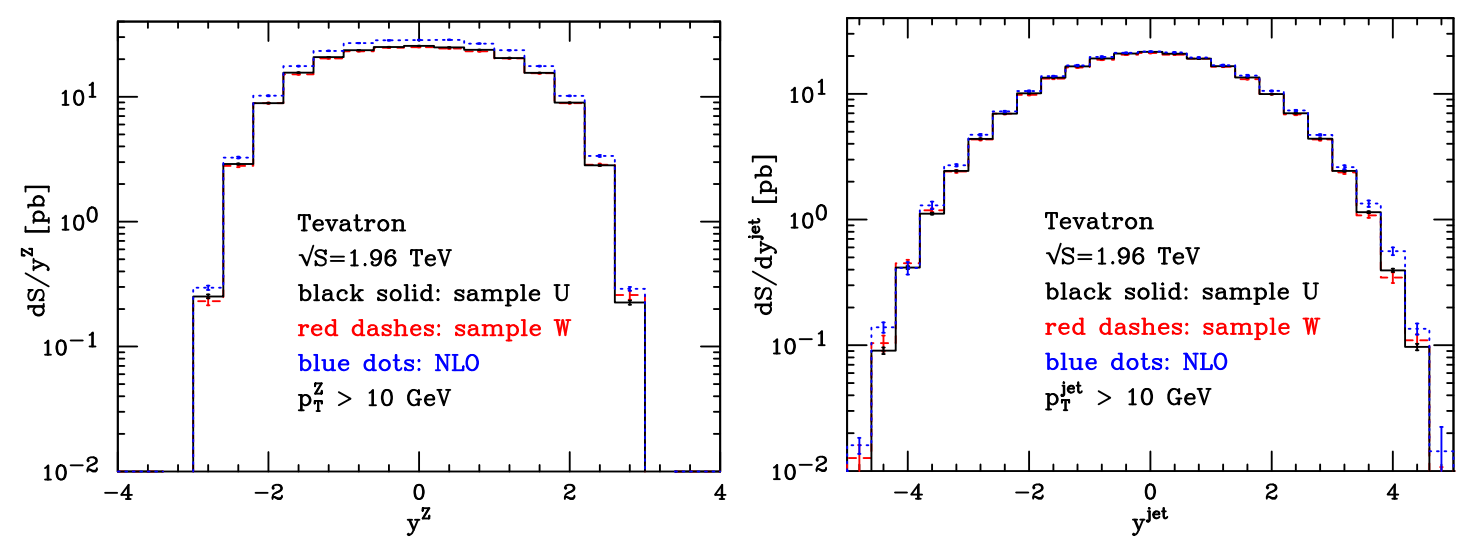

Figure 5. Rapidity distribution of the $Z$ and of the hardest jet in samples $\mathrm{U}$ and $\mathrm{W}$, with a $p_{\mathrm{T}}$ cut on the transverse momentum of the $Z /$ jet of $10 \mathrm{GeV}$.

parameters were introduced in the framework of the POWHEG implementation of heavy-quark pair production [17], and are mentioned in the corresponding manual [18], and discussed extensively in ref. [9]. We briefly recall their genesis and use in appendix B.

A clarifying consideration on negative weights in POWHEG can be made in comparison with MC@NLO [19]. The negative weights that we get in POWHEG are fully analogous to the negative weights that one gets in the $\mathcal{S}$ events in MC@NLO. Also in that case, the number of negative weights could be reduced or eliminated by using a folding technique, as in our case. However, negative weights in the $\mathcal{H}$ event sample of MC@NLO cannot be reduced, and so there is no reason to attempt to reduce the negative weight fraction in the $\mathcal{S}$ events.

Finally, let us stress again that by performing any analysis on a POWHEG event sample with signed events, or with weighted events, or with positive-weight events (with a large enough folding number to get a negligible fraction of negative weights) yields the same result within statistics. This property can be easily verified by running the program with powheg.input files that differ only for the presence of the withnegweights flag set to 1 , or for the presence of the bornsuppfact token, or, again, differing only in the folding numbers. In the current version of the POWHEG BOX we leave this choice to the user. The performance cost of getting positive weights may be well balanced if the events have to be run through costly detector simulators, or if it is undesirable to have negative weighted events in some complex, multivariate statistical analysis. The default POWHEG BOX setting is with no negative weights and no weighted events (i.e. $F\left(k_{\mathrm{T}}\right)=1$ ).

\subsection{Comparison with MCFM}

As a final check of the code, we have compared the NLO output of the POWHEG BOX to the output produced by the NLO code from MCFM [20], and total agreement was found. In spite of the fact that we have taken the virtual correction formulae (that we have checked against the BlackHat [21] results too) from the MCFM program, this comparison is a highly non-trivial check, since the subtraction schemes used by the two programs are completely different. 


\section{Phenomenology}

In this section we directly compare the output of our program to distributions that have been measured by the CDF and D0 Collaborations at the Tevatron. The only aim of this study is to validate, to some extent, our program. We believe that a more thorough analysis can only be performed by the experimental collaborations themselves, using our event generator. All the results displayed in this section have been obtained with a sample of roughly 1.3 million events, at the center-of-mass energy of $1.96 \mathrm{TeV}$, in proton-antiproton collisions. The sample was generated with positive weights, with the folding parameters 510-5 (see section 3.1). The generation cut was set to $5 \mathrm{GeV}$. The fraction of negative-weight events generated with this setting of parameters was $0.4 \%$ and these events were disregarded in the following analysis. As shown in section 3.1, this fraction is concentrated in events with low transverse momenta. We have used the CTEQ6M pdf set, with the corresponding value of $\Lambda_{\mathrm{QCD}}$. The renormalization and factorization scales for the calculation of the $\bar{B}$ function are set equal to the $Z$ transverse momentum in the underlying Born configuration.

The events were showered using PYTHIA 6.4.21 [22]. We have compared two different choices of tune for PYTHIA: the tune A (PYTUNE(100)), that uses the old shower and underlying event model, and the Perugia 0 tune (PYTUnE(320)), that uses the new transversemomentum ordered shower and the new underlying event model. Results for the Tune A model will be displayed as blue dashed histograms, while the Perugia 0 will be shown as red solid lines. The data will be displayed as simple points with error bars.

We have switched off photon radiation off leptons (mstj $(41)=3$ ), so that, in the case of $Z \rightarrow \ell^{+} \ell^{-}$, the lepton energy better represents what would be measured in an electromagnetic calorimeter. In addition we have set the $Z$ mass and width to the values $91.188 \mathrm{GeV}$ and $2.486 \mathrm{GeV}$ respectively, $\sin ^{2} \theta_{W}^{\mathrm{eff}}=0.2312$ and $\alpha_{\mathrm{em}}^{-1}\left(M_{Z}\right)=128.930$.

We applied the jet algorithm to all particles in the event, including all leptons, except for those coming from $Z$ decay. In other words, when comparing to experimental results, we assume that the jet energies are fully corrected to the particle level, including those particles that would not be visible in the detector. We have used the jet algorithms as implemented in the FAST JET package [23].

\subsection{CDF results}

The CDF Collaboration provided and still provides results for $Z / \gamma\left(\rightarrow e^{+} e^{-}\right)+1 j$ and $Z / \gamma\left(\rightarrow \mu^{+} \mu^{-}\right)+1 j$ events. In order to perform an analysis as similar as possible to the one done by the CDF Collaboration, we used the midpoint algorithm [24] to combine hadrons (from POWHEG events showered by PYTHIA) into jets, with cone radius $R=0.7$ and a merging/splitting fraction of 0.75 , starting from seed towers with transverse momenta above $1 \mathrm{GeV}$.

$Z / \gamma\left(\rightarrow e^{+} e^{-}\right)+$jets. We begin by considering the $Z / \gamma \rightarrow e^{+} e^{-}$results of CDF. In figures 6,7 and 8 we compare the POWHEG results showered by PYTHIA using Tune A (blue dashed lines) and Perugia 0 tuning (red solid lines), with the CDF data.

The results in figures 6 and 7 were published in [1], while those in figure 8 were extracted from the blessed results in [25], at an integrated luminosity of $2.5 \mathrm{fb}^{-1}$. 


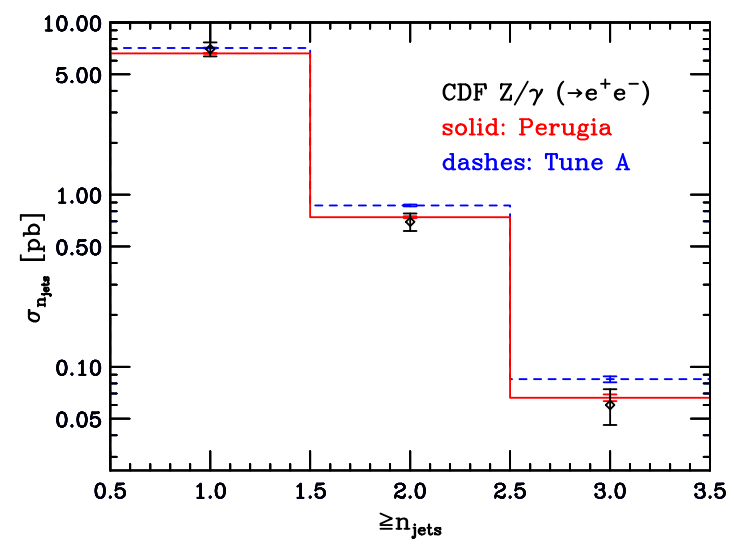

Figure 6. Total cross section for inclusive jet production.
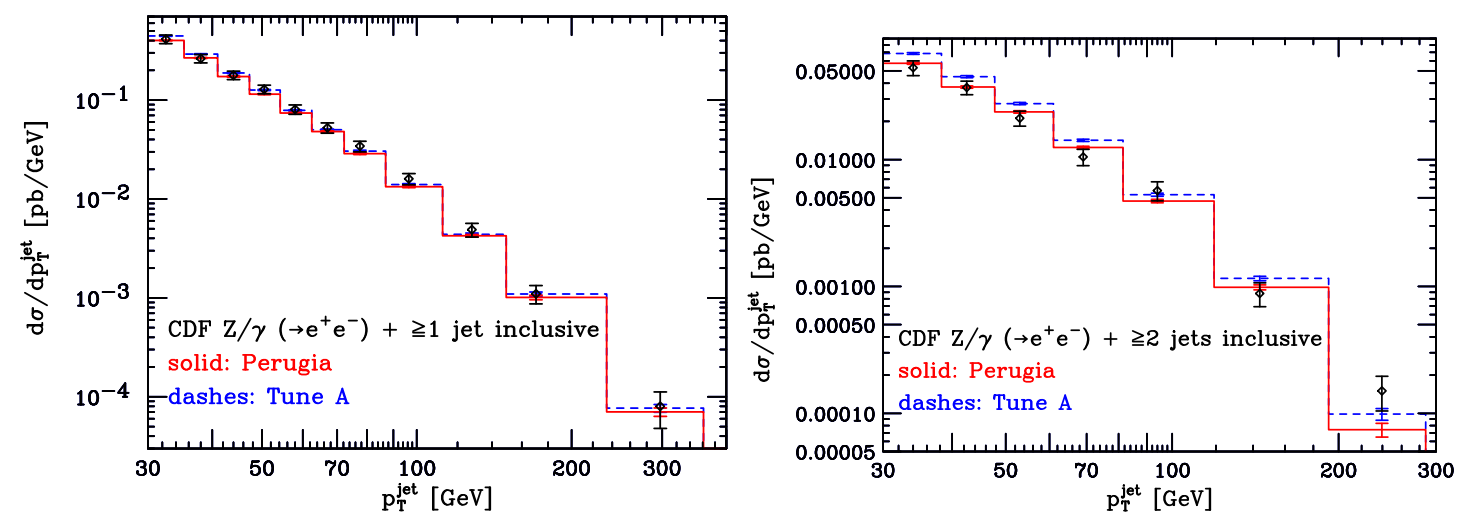

Figure 7. The inclusive $p_{\mathrm{T}}$ distributions for events with at least one and two jets.

In figures 6 and 7 we plot results for the inclusive total cross section and inclusive $p_{\mathrm{T}}$ distributions for the production of at least one and two jets. In figure 8 we plot the $p_{\mathrm{T}}$ distribution of the hardest and next-to-hardest jet and the inclusive rapidity distributions for events with at least one and two jets.

In order to compare with CDF data, we adopted the following cuts

$$
\begin{aligned}
& 66 \mathrm{GeV}<M_{e e}<116 \mathrm{GeV}, \quad p_{T}^{e}>25 \mathrm{GeV}, \quad\left|\eta^{e_{1}}\right|<1.0, \quad 1.2<\left|\eta^{e_{2}}\right|<2.8, \\
& \left|y^{\text {jet }}\right|<2.1, \quad \quad p_{T}^{\text {jet }}>30 \mathrm{GeV}, \quad \Delta R_{e, \text { jet }}>0.7,
\end{aligned}
$$

where $y$ and $\eta$ represent the rapidity and pseudorapidity of the specified particles, and where $R$ is the distance in the azimuth-rapidity plane.

We notice the good agreement between the POWHEG prediction and the data. It parallels the agreement between data and the NLO MCFM result displayed in refs. [1, 25], despite the fact that, when more than two jets are considered, MCFM has NLO accuracy, while our generator is limited to leading order. However, we emphasize that the POWHEG results are directly compared to data, while the MCFM ones are first corrected by parton-to-hadron correction factors, as detailed in [1]. Notice also the dependence of the results from the 

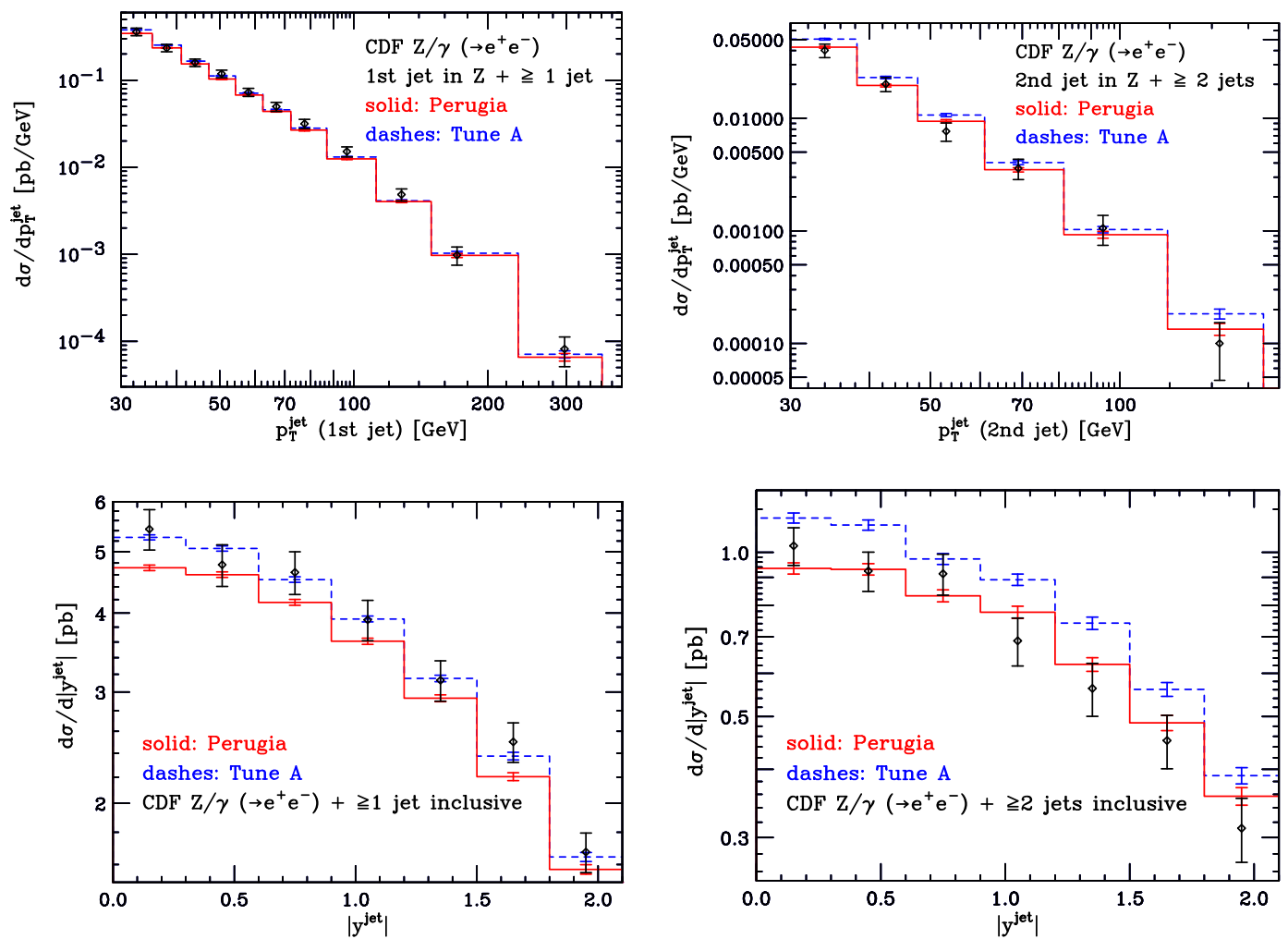

Figure 8. $p_{\mathrm{T}}$ distributions of the hardest and next-to-hardest jet and the inclusive rapidity distributions for events with at least one and two jets.

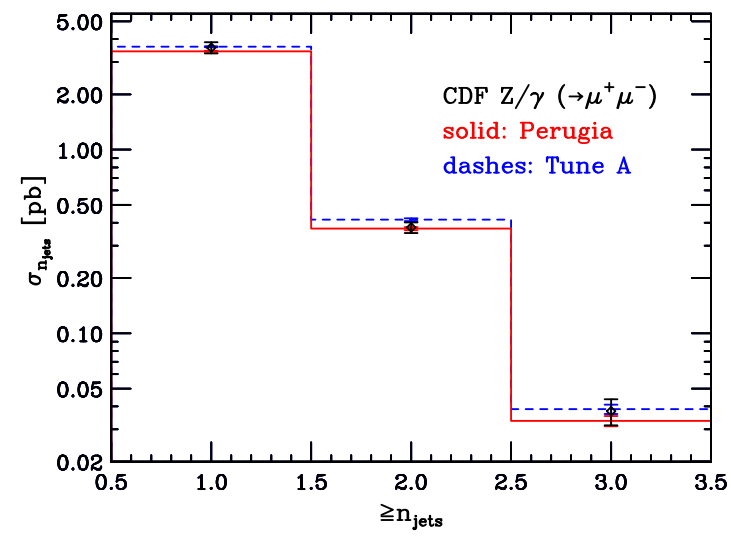

Figure 9. Total cross section for inclusive jet production.

chosen tune of PYTHIA. The Perugia 0 tune seems to give a slightly better agreement with data. We point out that the differences between the POWHEG results and the data is of the same order of the differences between the two tunes, thus suggesting that, by directly tuning the POWHEG results to data, one may get an even better agreement.

$Z / \gamma\left(\rightarrow \boldsymbol{\mu}^{+} \boldsymbol{\mu}^{-}\right)+$jets. Similar studies for the $Z / \gamma$ decaying in the $\mu^{+} \mu^{-}$channel were also performed by CDF. In figures 9 and 10 we display the total cross section for inclusive 

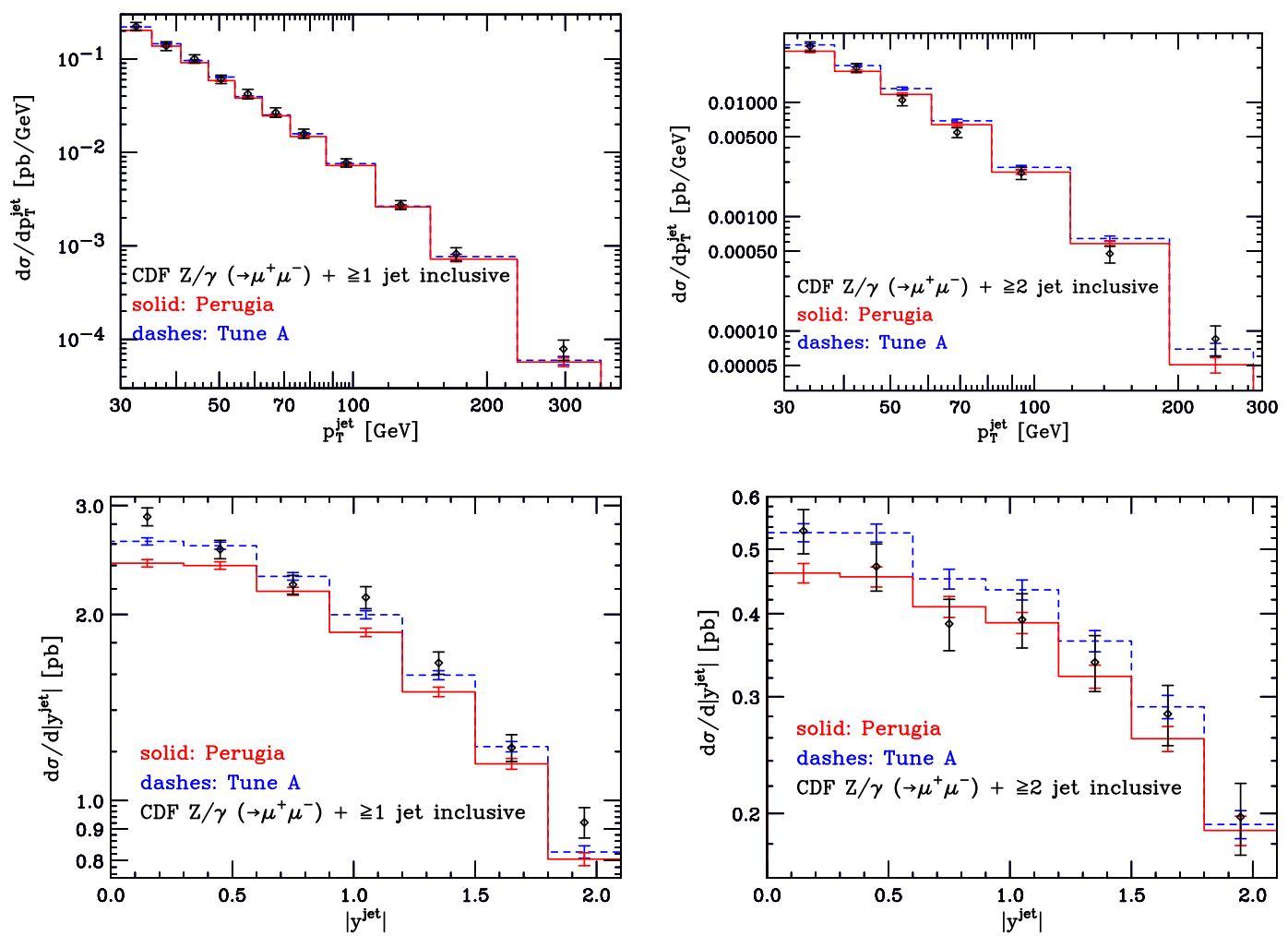

Figure 10. The inclusive $p_{\mathrm{T}}$ and rapidity distributions for events with at least one and two jets.

jet production and the inclusive $p_{\mathrm{T}}$ and rapidity distributions for events with at least one and two jets. In order to perform an analysis as close as possible to the CDF experimental settings, we have applied the following cuts

$$
\begin{aligned}
& 66 \mathrm{GeV}<M_{\mu \mu}<116 \mathrm{GeV}, \quad \quad p_{T}^{\mu}>25 \mathrm{GeV}, \quad\left|\eta^{\mu}\right|<1.0, \\
& \left|y^{\text {jet }}\right|<2.1, \quad \quad p_{T}^{\text {jet }}>30 \mathrm{GeV}, \quad \Delta R_{\mu, \text { jet }}>0.7 .
\end{aligned}
$$

In this case, no clear conclusions can be drawn on which of the two chosen tunes better reproduces the data.

\subsection{Do results}

The D0 Collaboration performed analyses similar to those done by the CDF Collaboration, focusing on more exclusive jet cross sections, and considering also some angular distributions.

To perform an analysis as similar as possible to the one done by the D0 Collaboration, we used the D0 Run II iterative seed-based cone jet algorithm [26] in order to recombine hadrons into jets, with a splitting/merging fraction of 0.5 and a cone radius $R=0.5$. 

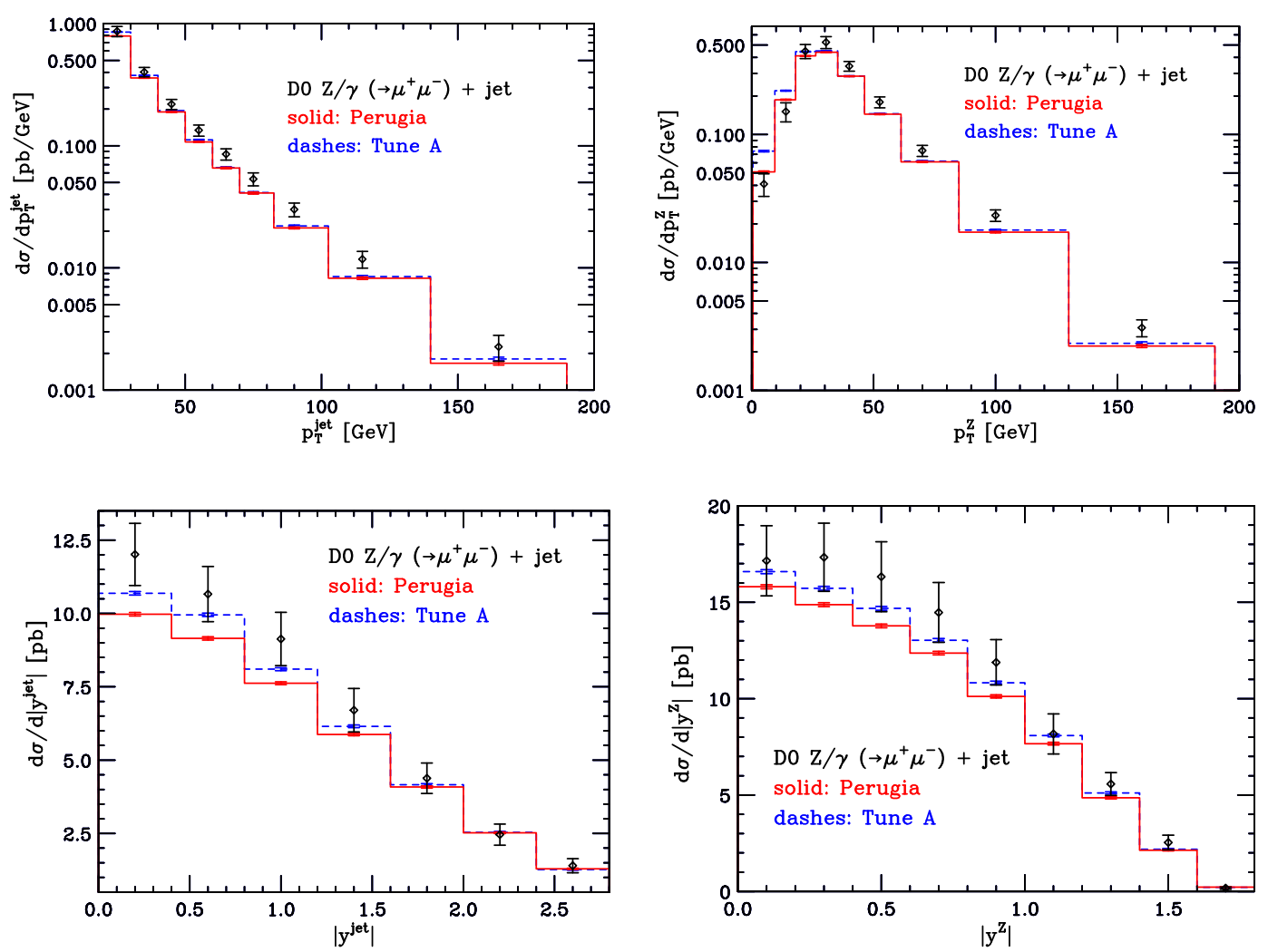

Figure 11. Distributions of the transverse momentum and rapidity of the hardest jet and of the $Z$ boson.

$Z / \gamma\left(\rightarrow \boldsymbol{\mu}^{+} \boldsymbol{\mu}^{-}\right)+$jets. In ref. [3, 5], several distributions were studied by the D0 experiment using the set of cuts

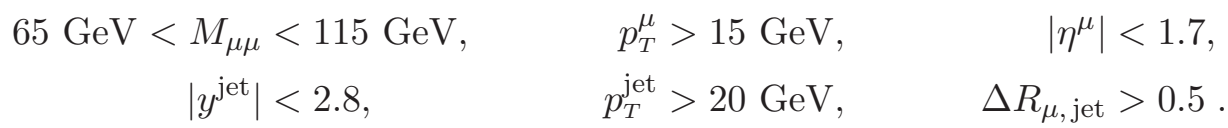

In figure 11, we plot distributions of the transverse momentum and rapidity of the hardest jet and of the $Z$ boson. In figure 12, we show the azimuthal separation and rapidity separation between the $Z$ boson and the leading jet, for events with $p_{T}^{Z}>25 \mathrm{GeV}$ and $p_{T}^{Z}>45 \mathrm{GeV}$, while in figure 13 we plot the absolute value of the average rapidity of the $Z$ and of the leading jet, $\mid y_{\text {boost }}(Z+$ jet $) \mid$, for events with $p_{T}^{Z}>25 \mathrm{GeV}$ and $p_{T}^{Z}>45 \mathrm{GeV}$, where

$$
y_{\text {boost }}(Z+\text { jet })=\frac{1}{2}\left(y^{Z}+y^{\text {jet }}\right),
$$

$y^{\text {jet }}$ being the rapidity of the leading jet.

In ref. [5], the D0 Collaboration plotted the normalized differential cross sections, in order to reduce the associated error bars. We have, instead, preferred to plot the differential cross sections itself, in order to appreciate how well POWHEG reproduce not only the shapes but also the overall normalization. The total cross section they use to normalize is given in [3] and is equal to

$$
\sigma_{Z}=118 \pm 0.5 \text { (stat.) } \pm 4 \text { (syst.) } \pm 4 \text { (muon) } \pm 7 \text { (lumi.) pb. }
$$



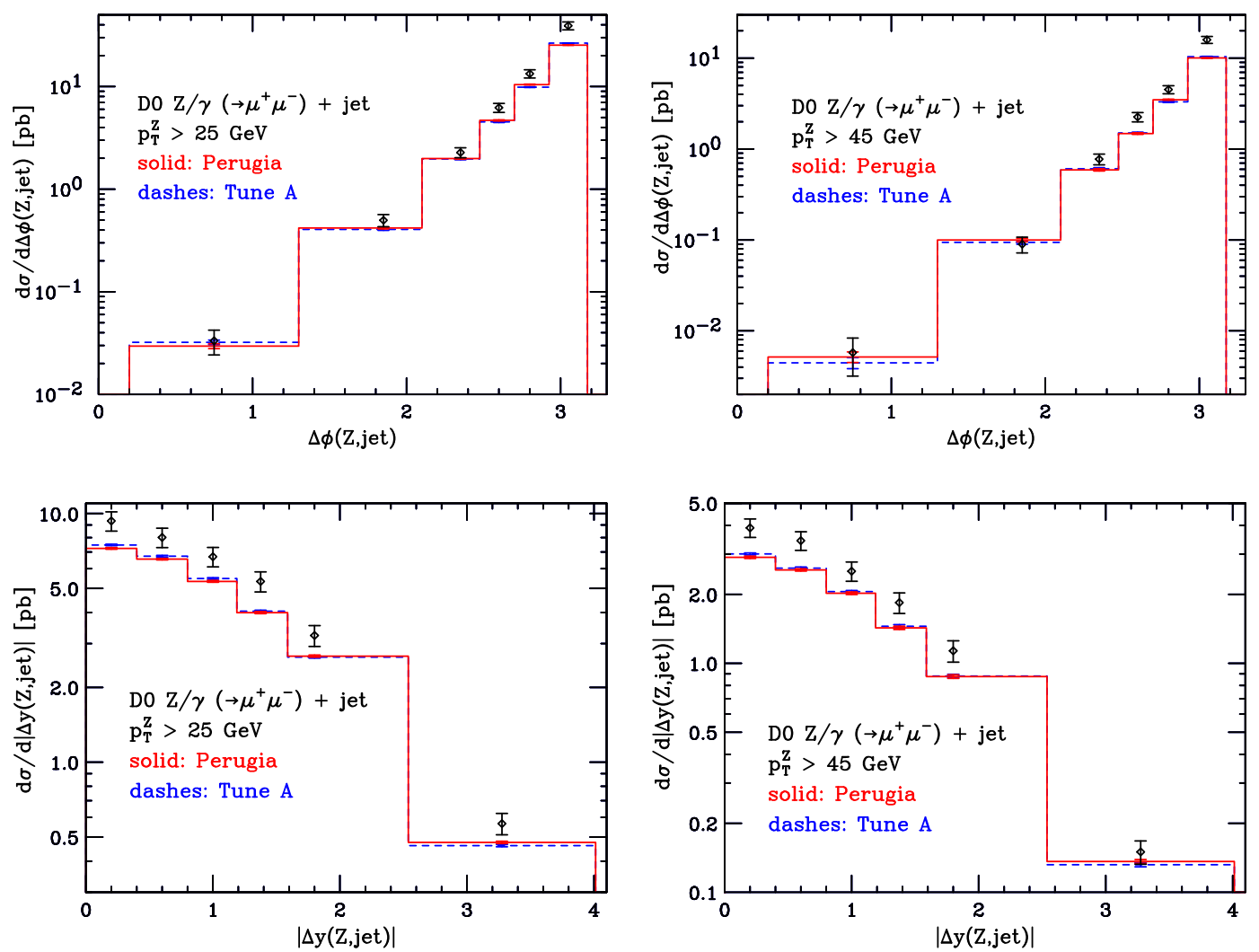

Figure 12. Azimuthal separation and rapidity separation between the $Z$ boson and the leading jet, for events with $p_{T}^{Z}>25 \mathrm{GeV}$ and $p_{T}^{Z}>45 \mathrm{GeV}$.
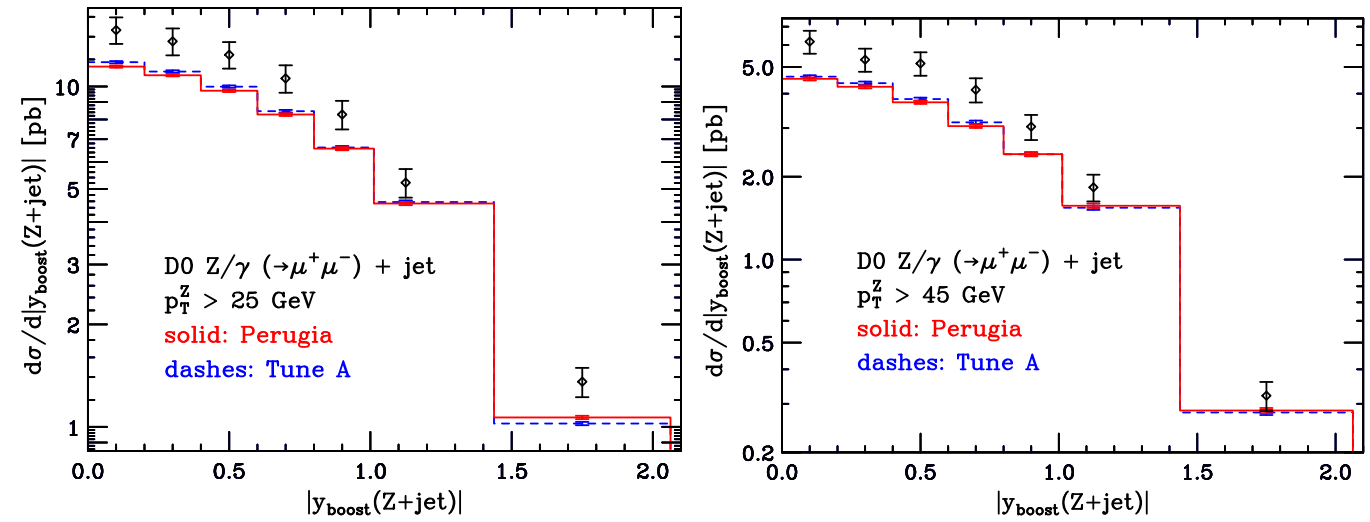

Figure 13. Absolute value of the average rapidity of the $Z$ and of the leading jet, $\mid y_{\text {boost }}(Z+$ jet $) \mid$, for events with $p_{T}^{Z}>25 \mathrm{GeV}$ and $p_{T}^{Z}>45 \mathrm{GeV}$.

In addition, we have added, in quadrature, a flat systematic luminosity error of $8 \%$ to every bin.

We see a noticeable discrepancy between data and the POWHEG prediction in the shape of the transverse-momentum spectra of the jet and of the $Z$. This discrepancy also manifest 

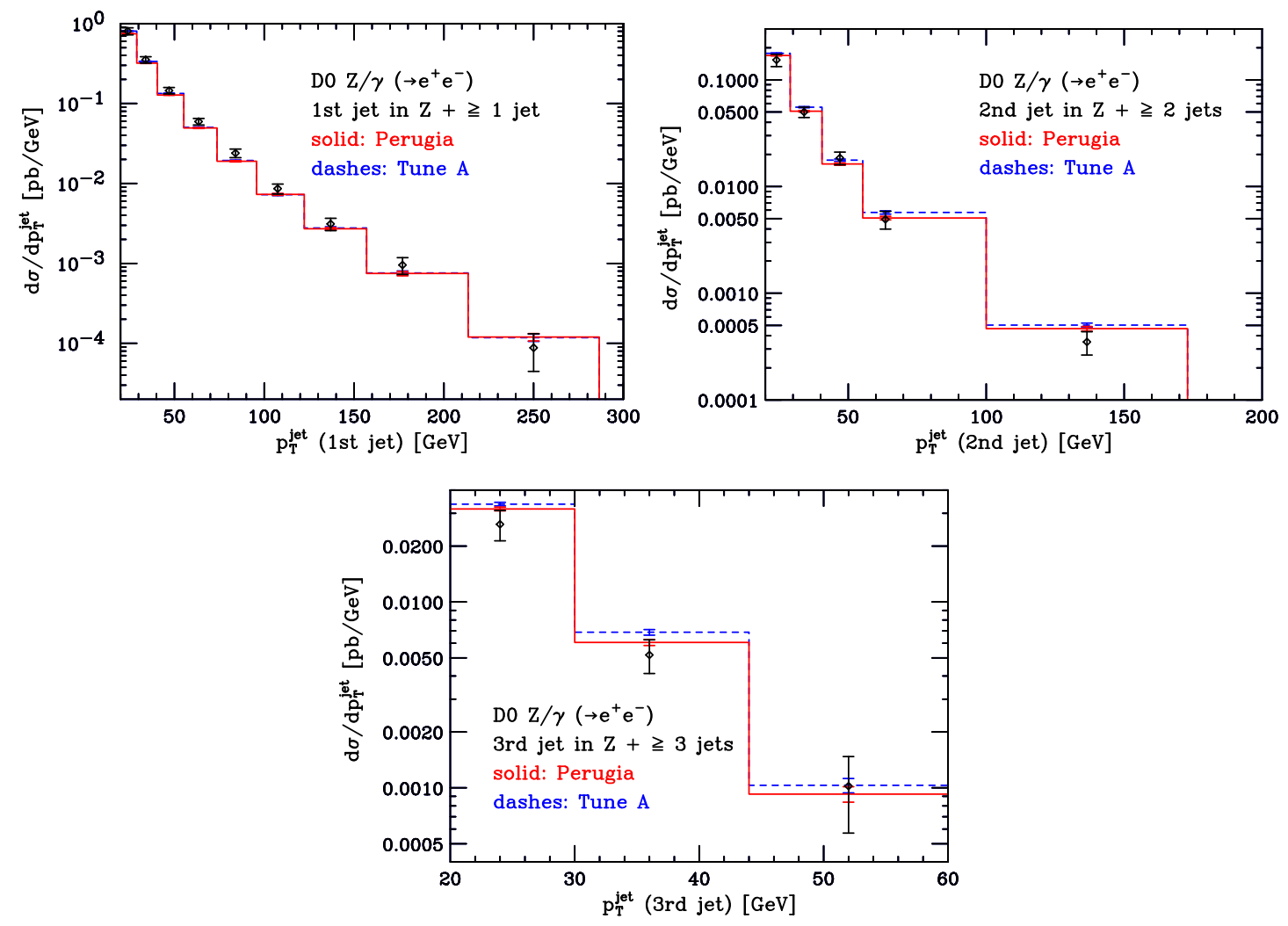

Figure 14. $p_{\mathrm{T}}$ distributions of the hardest, next-to-hardest and next-to-next-to-hardest jet.

itself in differences in the normalization of the angular distributions in figures 12 and 13, since transverse momentum cuts are applied there. We do not wish to comment further on this problem, that, on the other hand, is not present in the CDF analyses, and in the D0 analysis of the $Z / \gamma \rightarrow e^{+} e^{-}$channel. We remark that the problem may well be due to our failure to understand some features of the D0 analysis, rather than to problems in the data or in POWHEG. We also notice that the angular correlation between the $Z$ and the jet $p_{\mathrm{T}}$ is only qualitatively described by POWHEG. We remind the reader, however, that POWHEG has only LO accuracy for this quantity, that is determined by the emission of a second hard parton not included in the hardest jet, and that near the back-to-back region, Sudakov resummation effects, as well as non perturbative effects, become determinant.

$Z / \gamma\left(\rightarrow e^{+} e^{-}\right)+$jets. D0 has published studies for the $Z / \gamma\left(\rightarrow e^{+} e^{-}\right)+1 j$ channel in ref. [4]. In figure 14 we have plotted the $p_{\mathrm{T}}$ distributions of the hardest, next-to-hardest and next-to-next-to-hardest jet. No information is given in the paper on the value of the total inclusive $Z$ cross section used to normalized the distributions. It is reasonable to use the same total cross section of eq. (4.5).

The cuts that we have applied for this analysis were the following

$$
\begin{aligned}
65 \mathrm{GeV}<M_{e e} & <115 \mathrm{GeV}, & p_{T}^{e} & >25 \mathrm{GeV}, \\
\left|y^{\text {jet }}\right| & <2.5, & p_{T}^{\text {jet }} & >20 \mathrm{GeV} .
\end{aligned}
$$



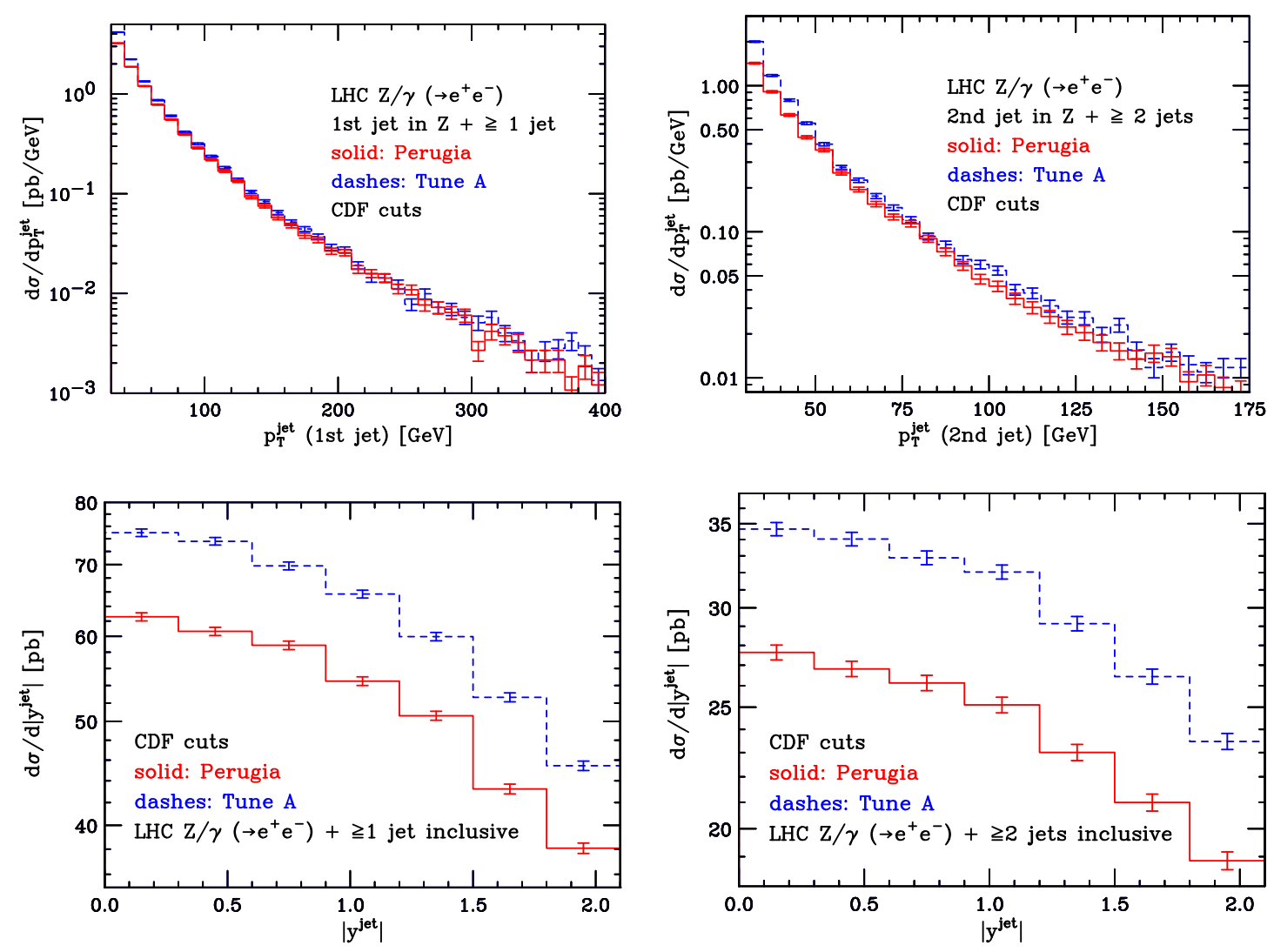

Figure 15. $p_{\mathrm{T}}$ distributions of the hardest and next-to-hardest jet and the inclusive rapidity distributions for events with at least one and two jets at the $\mathrm{LHC}$ at $14 \mathrm{TeV}$, with the cuts of eq. (4.1).

We can see quite a good agreement among the data and the two POWHEG distributions for the hardest and next-to-hardest jet. Unexpectedly, there is quite a good agreement for the next-to-next-to-hardest jet too: in fact, this jet is a shower jet, so we do not expect it to be correct away from the collinear limit.

\subsection{LHC results}

In this section, we show a few results for the LHC at $14 \mathrm{TeV}$. We have produced 1.400 .000 events, generated with the withnegweights flag set to 1 , with $k_{\text {gen }}=5 \mathrm{GeV}$ and with a 5-10-5 folding. With this folding, the fraction of negative weights is around $1.6 \%$. We have then showered the events generated by POWHEG with PYTHIA, using the tune A and the Perugia 0 tuning. In figures 15 and 16 we have plotted a few distributions obtained for the LHC, using the same cuts and the same jet algorithms used by the two Collaborations at the Tevatron: in figure 15, we have applied the cuts of eq. (4.1) that CDF used for the $Z / \gamma\left(\rightarrow e^{+} e^{-}\right)+1 j$ analysis, while in figure 16, we have applied the cuts of eq. (4.3), that D0 used for the $Z / \gamma\left(\rightarrow \mu^{+} \mu^{-}\right)+1 j$ study.

In figure 15 we can see a pattern of the two tunes similar to the one depicted in the corresponding figure 8: small differences for the distribution of the hardest jet, some 

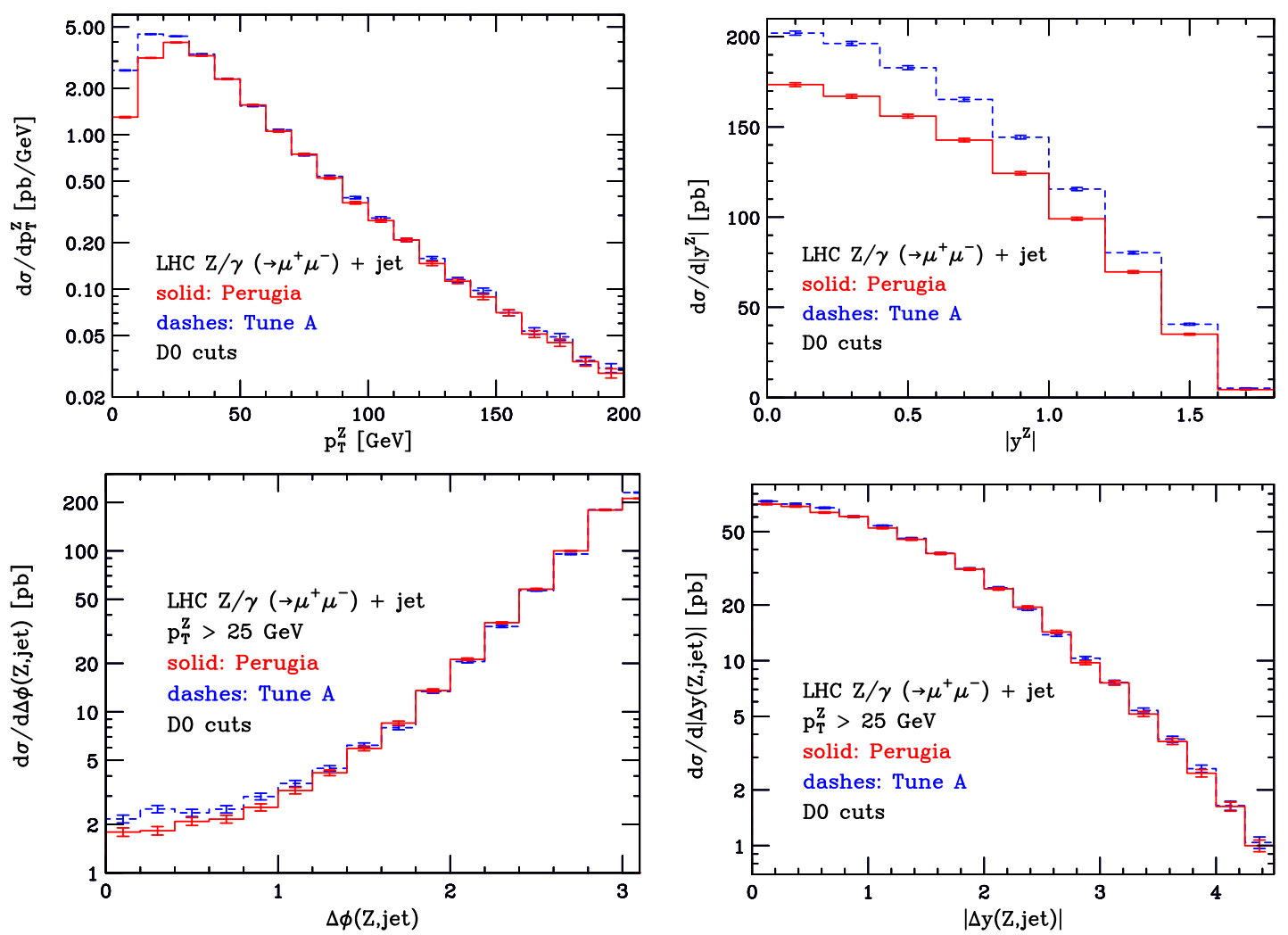

Figure 16. Distributions of the transverse momentum and rapidity of the $Z$ boson and azimuthal separation and rapidity separation between the $Z$ boson and the leading jet, for events with $p_{T}^{Z}>$ $25 \mathrm{GeV}$, at the LHC at $14 \mathrm{TeV}$, with the cuts of eq. (4.3).

differences both at low and high $p_{\mathrm{T}}$ for the next-to-hardest jet, and increasing differences in their inclusive rapidity distributions, with peaks of $\sim 20 \%$. Similarly, figure 16 should be compared with the corresponding panels in figures 11 and 12. Tune A gives a higher cross section at low transverse momentum and central rapidity of the vector boson with respect to the Perugia 0 tuning, while they agree very well in the azimuthal separation and rapidity separation between the $Z$ boson and the leading jet, for events with $p_{T}^{Z}>25 \mathrm{GeV}$.

\section{Conclusions}

In this paper, we have presented, for the first time, a calculation of vector boson plus one jet NLO cross section interfaced to a Shower Monte Carlo program, within the POWHEG framework. More specifically, we have implemented this process using the POWHEG BOX, a general computer code framework for embedding NLO calculations in shower Monte Carlo programs according to the POWHEG method.

We have compared the POWHEG results for $Z / \gamma+1 j$ with the data available from the CDF and the D0 Collaborations, using two different tunes for PYTHIA. We notice that differences between the POWHEG results and the data is of the same order of the differences 
between the two tunes, thus suggesting that, by directly tuning the POWHEG results to data, one may get an even better agreement.

Finally, we have presented similar results for the LHC, running at $14 \mathrm{TeV}$, showing that, in some distributions, the difference between the two tunes is of the order of $20 \%$.

The code of our generator can be accessed in the POWHEG BOX svn repository:

svn://powhegbox.mib.infn.it/trunk/POWHEG-BOX,

with username anonymous and password anonymous.

\section{Acknowledgments}

We thank John Campbell for helping us to access the virtual corrections in the MCFM code. We also thank Gavin Hesketh, Sabine Lammers and Henrik Nilsen for useful discussions on the experimental side. E.R. thanks Daniel Maître for his help comparing BlackHat and MCFM results. The work of S.A. has been supported in part by the Deutsche Forschungsgemeinschaft in SFB/TR 9. S.A. and E.R. would like to thank DESY Zeuthen for the use of the computing facilities and the hospitality, and CERN, where part of this work was performed.

\section{A Tracking negative-weight events}

In order to investigate what happens at small transverse momenta, where the NLO computation is no longer feasible, we have introduced a new feature in the POWHEG BOX, in order to track negative-weight events. This feature is activated by setting the token withnegweights to 1 (true). If withnegweights is set to 1 , events with negative weight can thus appear in the Les Houches event file [27, 28]. While we normally set the IDWTUP flag in the Les Houches interface to 3 , in this case we set it to -4 . With this flag, the SMC is supposed to simply process the event, without taking any other action. Furthermore, the XWGTUP (Les Houches) common block variable is set by the POWHEG BOX to the sign of the event times the integral of the absolute value of the cross section, in such a way that its average equals the true total cross section.

We preferred not to use the option IDWTUP $=-3$ for signed events with constant absolute value. This option is advocated by the Les Houches interface precisely in such cases, and it requires that the event weight XWGTUP assumes the values \pm 1 . However, the Les Houches interface does not provide a standard way to store the integral of the absolute value of the cross section, that would be needed to compute correctly the weight of the event in this case. In fact, the XSECUP variable is reserved for the true total cross section $\sigma_{\mathrm{NLO}}$. More specifically, suppose that the total NLO cross section receives contributions from regions in the phase space where the differential cross section is positive, and where it is negative, so that we can write

$$
\sigma_{\mathrm{NLO}}=\sigma_{(+)}-\left|\sigma_{(-)}\right| .
$$

We then generate a sample of $N=N_{+}+N_{-}$, with $N_{+}$events with weight $W_{i}=+1$ (so that the sum of the $W_{i}$ on events with positive weights is $N_{+}$) and $N_{-}$events with weight 
$W_{i}=-1$ (so that the sum of the $W_{i}$ on events with negative weights is $-N_{-}$) in such a way that

$$
N_{+}=\frac{\sigma_{(+)}}{\sigma_{(+)}+\left|\sigma_{(-)}\right|} N, \quad N_{-}=\frac{\sigma_{(-)}}{\sigma_{(+)}+\left|\sigma_{(-)}\right|} N .
$$

In order to give the correct NLO cross section, the $N$ events should then be weighted with the sum of the positive plus the absolute value of the negative part of the cross section, in such a way that

$$
\frac{1}{N} \sum_{i=1}^{N} W_{i}\left(\sigma_{(+)}+\left|\sigma_{(-)}\right|\right)=\sigma_{(+)}-\left|\sigma_{(-)}\right|=\sigma_{\mathrm{NLO}} .
$$

Summarizing, since there is no standard way in the Les Houches interface to pass this absolute value when IDWTUP $=-3$, we set it to -4 , and set the weight XWGTUP of the $i$-th event to $W_{i} \times\left(\sigma_{(+)}+\left|\sigma_{(-)}\right|\right)$, so that, event by event, we have this information. In this case the average value of the XWGTUP variable is equal to the total cross section, as required by the Les Houches interface when IDWTUP $=-4$.

Notice that, if withnegweights is set to true and a Born suppression factor is also present, the events will have variable XWGTUP of either signs. In this case, XWGTUP is set to the sign of the event, times the absolute value of the cross section, divided by the suppression factor (the output of the born_suppression routine). Also in this case, the average value of XWGTUP coincides with the true total cross section. Weighted events are also useful if one wants to generate a homogeneous sample from relatively-low up to veryhigh transverse momenta. In this case, it is convenient to pick a very large bornsuppfact value, of the order of the maximum transverse momentum one is interested in. The largemomentum region will be more populated in this way. The form of the born_suppression function can also be changed at will by the user.

\section{B Negative weights and folding}

In POWHEG, the underlying Born configuration for an event is generated according to the $\bar{B}$ function, that, in the simplest cases, can be represented by the equation (see ref. [8])

$$
\bar{B}\left(\Phi_{B}\right)=B\left(\Phi_{B}\right)+V\left(\Phi_{B}\right)+\int d \Phi_{\mathrm{rad}}\left[R\left(\Phi_{B}, \Phi_{\mathrm{rad}}\right)-C\left(\Phi_{B}, \Phi_{\mathrm{rad}}\right)\right] .
$$

In order to generate $\Phi_{B}$ distributed according to this probability density, we actually parametrize the radiation variables in terms of three variables in the unit cube, $X_{i}$, and then perform the integral

$$
\int d \Phi_{B} d^{3} X \tilde{B}\left(\Phi_{B}, X\right)
$$

where

$$
\tilde{B}\left(\Phi_{B}, X\right)=B\left(\Phi_{B}\right)+V\left(\Phi_{B}\right)+\left|\frac{\partial \Phi_{\mathrm{rad}}}{\partial X}\right|\left[R\left(\Phi_{B}, \Phi_{\mathrm{rad}}\right)-C\left(\Phi_{B}, \Phi_{\mathrm{rad}}\right)\right],
$$

using the MINT integration program [29]. This program stores appropriate grids and upper bounds, so that, after the integration, it is possible to use it to generate points in the 
integration variables, i.e. in $\left(\Phi_{B}, X\right)$, distributed with a probability proportional to $\tilde{B}$. The $X$ values are ignored, which amounts to integrating over them, and only $\Phi_{B}$ is retained. This procedure is sufficient to generate positive weights if the process in question has a sufficiently-high scale, like in vector boson, Higgs or $t \bar{t}$ production. In these cases, $\alpha_{\mathrm{S}}$ (present in eq. (B.3) in the virtual $V$, real $R$ and counterterms $C$ contributions) is small enough so that $B$ is always larger than the other terms, yielding a positive result for any value of $\Phi_{B}$ and $X$. As the scale of the process decreases (and $\alpha_{\mathrm{S}}$ increases), it becomes more likely that $\tilde{B}$ may become negative for some value of the $X$ parameters, even if $\bar{B}$ is positive for any value of $\Phi_{B}$. Of course, this depends upon the way that the $C$ counterterm is defined. A brute force remedy to this problem, is to fold the integration of the $(R-C)$ term as many time as necessary to yield a positive $\bar{B}$ (that is to say, to get a negligible fraction of negative-weight events). The details of the folding method are illustrated in the MINT manual. Here we only illustrate it with an example. If we want to fold the $X_{1}$ variable twice, we define

$$
\tilde{B}_{\text {folded }}\left(\Phi_{B}, x_{1}, X_{2}, X_{3}\right)=\tilde{B}\left(\Phi_{B}, x_{1}, X_{2}, X_{3}\right)+\tilde{B}\left(\Phi_{B}, 1 / 2+x_{1}, X_{2}, X_{3}\right),
$$

and integrate in $x_{1}$ from 0 to $1 / 2$. This clearly yields the same result as without the folding. Furthermore, if we increase the number of foldings for all the three parameters $X_{i}$, the folded function becomes less and less dependent upon the $x_{i}$ values. With this technique, regions of integration where the function is positive and negative are combined together. It is clear that, if the $\bar{B}$ function is positive, with large enough folding numbers, we will achieve the positivity of the folded $\tilde{B}$ function.

The MINT integrator and the POWHEG BOX can perform the folding automatically. The user needs only to specify how many times each variables should be folded. The MINT integrator divides each integration coordinates in 50 bins, such that each bin contributes equally to the integral of the absolute value of the $\tilde{B}$ function. Folding is achieved by overlapping these intervals. Thus, the folding number must be a divisor of 50: 2, 5, 10, 25 or 50. Three tokens in the powheg. input file can be set to the folding number: ifoldcsi, ifoldy and ifoldphi, that refer to the folding of the three radiation variables $\xi, y$ and $\phi$.

In figure 17, we display the effect of folding on the amount of negative weights that enter the computation of a physical quantity, namely the $Z$ transverse momentum (by choosing similar variables, like the transverse momentum of the hardest jet, we get similar results). All the histograms in the figure were obtained with the withnegweights flag set to true. The solid line was obtained in the standard way, i.e. by adding positive and negative contributions. The dashed, dotted and dotdashed lines were obtained by plotting only the absolute value of the negative weights. The dashed line was obtained with no folding, the dotted line was obtained with a 2-5-1 folding (i.e. ifoldcsi $=2$, ifoldy $=5$ and ifoldphi=1), and the dot-dashes line was obtained with the 5-10-5 folding. From the figure, we see that the amount of negative weights increases as the transverse momentum becomes smaller. This can be understood as being due to the increase of $\alpha_{\mathrm{S}}$ at small scales. By increasing the folding numbers, the number of negative weights is strongly reduced, and, furthermore, it only affects the region of very small transverse momenta, where the calculation becomes however unreliable. 


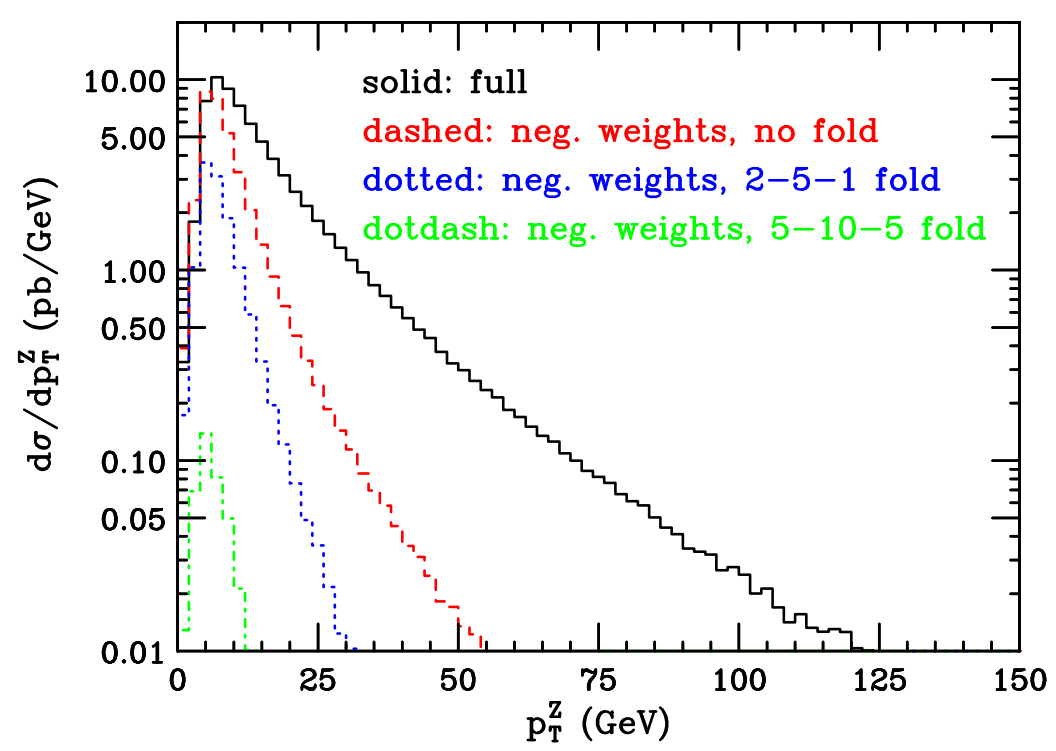

Figure 17. The effect of folding on the negative-weight event fraction.

\begin{tabular}{|l|c|c|c|c|c|}
\hline \multicolumn{1}{|c|}{$\xi$ - $y$ - $\phi$ folding $\Longrightarrow$} & $1-1-1$ & $2-5-2$ & $5-5-5$ & $5-10-5$ & $10-10-10$ \\
\hline $\mathrm{LHC}, 14 \mathrm{TeV}, k_{\text {gen }}=5 \mathrm{GeV}$ & 0.25 & 0.067 & 0.026 & 0.016 & 0.0074 \\
\hline $\mathrm{LHC}, 14 \mathrm{TeV}, k_{\text {gen }}=10 \mathrm{GeV}$ & 0.17 & 0.020 & 0.0029 & 0.00036 & $3.4 \times 10^{-5}$ \\
\hline Tevatron, $1.96 \mathrm{TeV}, k_{\text {gen }}=5 \mathrm{GeV}$ & 0.23 & 0.029 & 0.0086 & 0.0043 & 0.0028 \\
\hline
\end{tabular}

Table 1. Negative-weight fractions $\sigma_{(-)} /\left(\sigma_{(+)}+\left|\sigma_{(-)}\right|\right)$for given folding numbers.

Increasing the folding number has a cost on the execution time. As a rule of thumb, we expect the execution time to increase as the product of the three folding numbers. Actually, the increase is somewhat less than that, because the Born and virtual contribution are evaluated only once for each set of foldings, since they do not depend upon the integration variables. Thus, the folding should be chosen as a function of the generation cut, and, ultimately, as a function of the cutoff one is imposing on the hardest-jet transverse energy or on the $Z$ transverse momentum.

As an example, in table 1 , we have collected the fraction of negative weights $\sigma_{(-)} /\left(\sigma_{(+)}+\left|\sigma_{(-)}\right|\right)$for the LHC at $14 \mathrm{TeV}$, with two generation cuts, and for the Tevatron. As can be seen, the higher is the folding, the smaller is the fraction of negativeweight events.

The typical performance of event generation is, without folding, roughly 1000 events per minute, on typical workstation cpu's. It is clear that, in the worse case of very low transverse-momentum jets, assuming that we can tolerate around $2 \%$ negative weights (that would populate, in all cases, the very small $p_{\mathrm{T}}$ region), by using a 5-5-5 folding we end up generating of the order of eight events per minute. It is also clear that we have a compelling reason to generate independently samples with different generation cuts. Besides benefiting of a more abundant generation in the high $p_{\mathrm{T}}$ region, we also benefit from the possibility of using smaller folding numbers. 
Open Access. This article is distributed under the terms of the Creative Commons Attribution Noncommercial License which permits any noncommercial use, distribution, and reproduction in any medium, provided the original author(s) and source are credited.

\section{References}

[1] CDF - Run II collaboration, T. Aaltonen et al., Measurement of inclusive jet cross-sections in $Z / \gamma^{*}\left(\rightarrow e^{+} e^{-}\right)+$jets production in pp collisions at $\sqrt{s}=1.96 \mathrm{TeV}$, Phys. Rev. Lett. 100 (2008) 102001 [arXiv:0711.3717] [SPIRES].

[2] D0 collaboration, V.M. Abazov et al., Measurement of the ratios of the $Z / G^{*}+\geq n$ jet production cross sections to the total inclusive $Z / G^{*}$ cross section in p $\bar{p}$ collisions at $\sqrt{(s)}=1.96 \mathrm{TeV}$, Phys. Lett. B 658 (2008) 112 [hep-ex/0608052] [SPIRES].

[3] D0 collaboration, V.M. Abazov et al., Measurement of differential $Z / \gamma^{*}+$ jet $+X$ cross sections in pp collisions at $\sqrt{s}=1.96 \mathrm{TeV}$, Phys. Lett. B 669 (2008) 278 [arXiv:0808.1296] [SPIRES].

[4] D0 collaboration, V.M. Abazov et al., Measurements of differential cross sections of $Z / \gamma^{*}+$ jets $+X$ events in proton anti-proton collisions at $\sqrt{s}=1.96 \mathrm{TeV}$,

Phys. Lett. B 678 (2009) 45 [arXiv:0903.1748] [SPIRES].

[5] D0 collaboration, V.M. Abazov et al., Measurement of $Z / \gamma^{*}+j e t+X$ angular distributions in $p \bar{p}$ collisions at $\sqrt{s}=1.96 \mathrm{TeV}$, Phys. Lett. B $682(2010) 370$ [arXiv:0907.4286] [SPIRES].

[6] C. Buttar et al., Standard model handles and candles working group: tools and jets summary report, arXiv: 0803.0678 [SPIRES].

[7] P. Nason, A new method for combining NLO QCD with shower Monte Carlo algorithms, JHEP 11 (2004) 040 [hep-ph/0409146] [SPIRES].

[8] S. Frixione, P. Nason and C. Oleari, Matching NLO QCD computations with parton shower simulations: the POWHEG method, JHEP 11 (2007) 070 [arXiv: 0709. 2092] [SPIRES].

[9] S. Alioli, P. Nason, C. Oleari and E. Re, A general framework for implementing NLO calculations in shower Monte Carlo programs: the POWHEG BOX, JHEP 06 (2010) 043 [arXiv: 1002 .2581] [SPIRES].

[10] K. Hagiwara and D. Zeppenfeld, Helicity amplitudes for heavy lepton production in $e^{+} e^{-}$ annihilation, Nucl. Phys. B 274 (1986) 1 [SPIRES].

[11] K. Hagiwara and D. Zeppenfeld, Amplitudes for multiparton processes involving a current at $e^{+} e^{-}, e^{ \pm} p$ and hadron colliders, Nucl. Phys. B 313 (1989) 560 [SPIRES].

[12] J.M. Campbell and R.K. Ellis, Next-to-leading order corrections to $W^{+} 2$ jet and $Z^{+} 2$ jet production at hadron colliders, Phys. Rev. D 65 (2002) 113007 [hep-ph/0202176] [SPIRES].

[13] Z. Bern, L.J. Dixon and D.A. Kosower, One-loop amplitudes for $e^{+} e^{-}$to four partons, Nucl. Phys. B 513 (1998) 3 [hep-ph/9708239] [SPIRES].

[14] W.T. Giele and E.W.N. Glover, Higher order corrections to jet cross-sections in $e^{+} e^{-}$ annihilation, Phys. Rev. D 46 (1992) 1980 [SPIRES].

[15] S. Alioli, P. Nason, C. Oleari and E. Re, NLO vector-boson production matched with shower in POWHEG, JHEP 07 (2008) 060 [arXiv: 0805.4802] [SPIRES]. 
[16] J. Pumplin et al., New generation of parton distributions with uncertainties from global QCD analysis, JHEP 07 (2002) 012 [hep-ph/0201195] [SPIRES].

[17] S. Frixione, P. Nason and G. Ridolfi, A positive-weight Next-to-Leading-Order Monte Carlo for heavy flavour hadroproduction, JHEP 09 (2007) 126 [arXiv:0707.3088] [SPIRES].

[18] S. Frixione, P. Nason and G. Ridolfi, The POWHEG-hvq manual version 1.0, arXiv:0707.3081 [SPIRES].

[19] S. Frixione and B.R. Webber, Matching NLO QCD computations and parton shower simulations, JHEP 06 (2002) 029 [hep-ph/0204244] [SPIRES].

[20] J. Campbell and K. Ellis, MCFM - Monte Carlo for FeMtobarn processes, http://mcfm.fnal.gov.

[21] C.F. Berger et al., An automated implementation of on-shell methods for one-loop amplitudes, Phys. Rev. D 78 (2008) 036003 [arXiv:0803.4180] [SPIRES].

[22] T. Sjöstrand, S. Mrenna and P.Z. Skands, PYTHIA 6.4 physics and manual, JHEP 05 (2006) 026 [hep-ph/0603175] [SPIRES].

[23] M. Cacciari and G.P. Salam, Dispelling the $N^{3}$ myth for the $k_{t}$ jet-finder, Phys. Lett. B 641 (2006) 57 [hep-ph/0512210] [SPIRES].

[24] CDF collaboration, A. Abulencia et al., Measurement of the inclusive jet cross section in $p \bar{p}$ interactions at $\sqrt{s}=1.96 \mathrm{TeV}$ using a cone-based jet algorithm, Phys. Rev. D 74 (2006) 071103 [hep-ex/0512020] [SPIRES].

[25] CDF Run II QCD group results, http://www-cdf.fnal.gov/physics/new/qcd/QCD.html.

[26] G.C. Blazey and et al., Run II Jet physics in QCD and weak boson physics in Run II, FERMILAB-PUB-00-297 [SPIRES].

[27] E. Boos et al., Generic user process interface for event generators, hep-ph/0109068 [SPIRES].

[28] J. Alwall et al., A standard format for Les Houches event files, Comput. Phys. Commun. 176 (2007) 300 [hep-ph/0609017] [SPIRES].

[29] P. Nason, MINT: a computer program for adaptive Monte Carlo integration and generation of unweighted distributions, arXiv:0709.2085 [SPIRES]. 